\title{
Combined Experimental and Molecular Simulation Study of Insulin- 2Chitosan Complexation Driven by Electrostatic Interactions
}

\author{
3 Cecilia Prudkin-Silva, ${ }^{\dagger \oplus}$ Oscar E. Pérez, ${ }^{\dagger \oplus}$ Karina D. Martínez, ${ }^{\ddagger \odot}$ \\ 4 and Fernando L. Barroso da Silva*, ${ }^{*}, \| \odot$ \\ $5{ }^{\dagger}$ Departamento de Química Biológica, Facultad de Ciencias Exactas y Naturales, Instituto de Química Biológica de la Facultad de \\ 6 Ciencias Exactas y Naturales, IQUIBICEN-CONICET, Universidad de Buenos Aires, Buenos Aires, Intendente Güiraldes, s/n, \\ 7 Ciudad Universitaria, Pabellón 2, Buenos Aires CP 1428, Argentina \\ ${ }_{8}{ }^{\star}$ Departamento de Industrias, Facultad de Ciencias Exactas y Naturales, Consejo Nacional de Investigación Científica y Técnicas de \\ 9 la República Argentina, ITAPROQ-CONICET, Universidad de Buenos Aires, Intendente Güiraldes, s/n, Ciudad Universitaria, \\ 10 Buenos Aires CP 1428, Argentina \\ $11{ }^{\S}$ Departamento de Física e Química, Faculdade de Ciências Farmacêuticas de Ribeirão Preto, Universidade de São Paulo, Ribeirão \\ 12 Preto, São Paulo, 05508-220 Brazil \\ 13 "Department of Chemical and Biomolecular Engineering, North Carolina State University, Raleigh, North Carolina 27695, United \\ 14 States
}

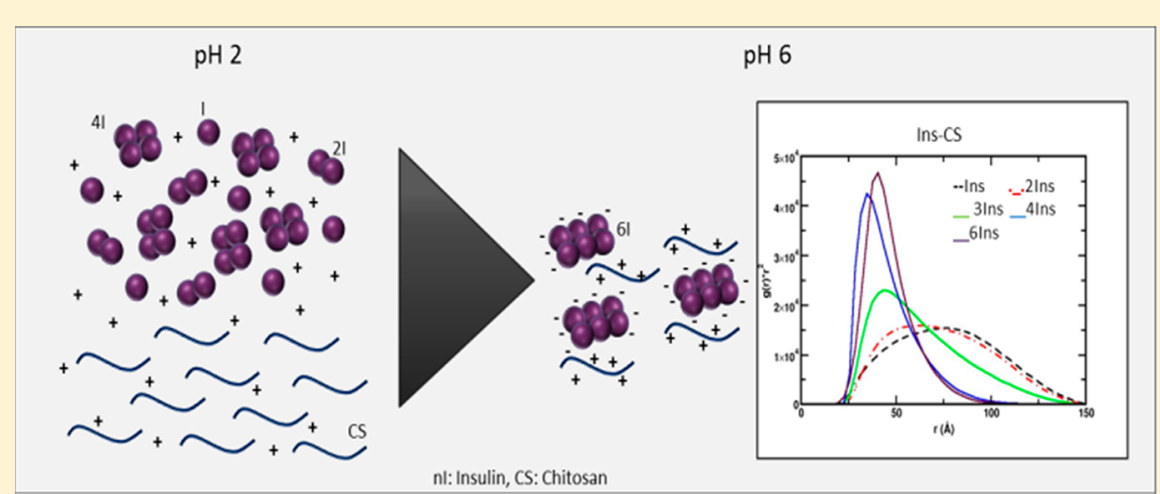

16 ABSTRACT: Protein-polysaccharide complexes constructed via self-assembly methods are often used to develop novel 17 biomaterials for a wide range of applications in biomedicine, food, and biotechnology. The objective of this work was to 18 investigate theoretically and to demonstrate via constant-pH Monte Carlo simulations that the complexation phenomenon 19 between insulin (INS) and the cationic polyelectrolyte chitosan (CS) is mainly driven by an electrostatic mechanism. 20 Experimental results obtained from FTIR spectra and $\zeta$-potential determinations allowed us to complement the conclusions. 21 The characteristic absorption bands for the complexes could be assigned to a combination of signals from CS amide I and INS 22 amide II. The second peak corresponds to the interaction between the polymer and the protein at the level of amide II. INS23 CS complexation processes not expected when INS is in its monomeric form, but for both tetrameric and hexameric forms, 24 incipient complexation due to charge regulation mechanism took place at $\mathrm{pH} 5$. The complexation range was observed to be 5.5 $25<\mathrm{pH}<6.5$. In general, when the number of INS units increases in the simulation process, the solution $\mathrm{pH}$ at which the 26 complexation can occur shifts toward acidic conditions. CS's chain interacts more efficiently, i.e. in a wider pH range, with INS 27 aggregates formed by the highest monomer number. The charge regulation mechanism can be considered as a previous phase 28 toward complexation (incipient complexation) caused by weak interactions of a Coulombic nature.

\section{INTRODUCTION}

${ }_{29}$ Over the last years, much attention has been focused on $\mathrm{pH}$ 30 responsive macromolecular mechanisms involved in protein31 polysaccharide interactions, as a tool for designing novel 32 structures with new functionalities. ${ }^{1}$ Thus, protein-polysac33 charide complexes constructed via self-assembly method were 34 often used as building blocks, to develop novel biomaterials for 35 a wide range of applications in biomedicine, food, and biotechnology. ${ }^{2,3}$ Self-assembly is a ubiquitous and natural 36 process that lead to complex nanostructures with outstanding 37 functionalities. ${ }^{4}$ In particular, the implementation of $\mathrm{pH}-38$

Special Issue: Molecular Simulation in Latin America: Coming of Age

Received: September 19, 2019

Published: December 2, 2019 
39 dependent responsive mechanisms was proven to be useful for 40 fabrication of pharmaceutical products resembling natural 41 protein assemblies. $^{5}$

42 Several types of proteins and polysaccharides have been used 43 for complexation. For example, Palao-Suay and collaborators 44 describe the use of self-assembling polysaccharides such as 45 dextran, chitosan, heparin, and alginate conjugated with 46 anticancer drugs such as doxorubicin, camptothecin, and 47 methotrexate. ${ }^{6}$ In regard to the food industry, it is a common 48 treatment to use proteins to ensure metabolic regulations for 49 beverage/solid applications, such as the soluble whey protein50 pectin complex as examined by Wagoner and Foegeding 51 (2017). ${ }^{2}$ This is a topic of high research activity in Latin 52 American countries. 4,10,19,24,25

53 In relation to the pharmaceutical industry, the use and 54 interactions of insulin (INS) and chitosan (CS) have also been 55 well reported in the literature. ${ }^{7-10}$ INS is a peptide hormone 56 produced by the pancreas that regulates glucose levels in the 57 bloodstream. Type I diabetes arises when INS is not produced, 58 and type II arises when production is scarce or the organism 59 rejects it. In both cases (I and II) it is difficult for glucose to 60 enter the cells, causing multiple adverse health effects. ${ }^{11}$ INS is 61 a therapeutically active protein and is used in the treatment of 62 diabetes mellitus at the recommended dose of $\geq 0.4 \mathrm{U} / \mathrm{kg}^{12}$ 63 Two polypeptide chains form INS: chain A consists of 21 64 amino acids, and chain B contains 30 amino acids. Chains A 65 and $B$ are held together by two disulfide $(S-S)$ bridges. In 66 addition, chain $\mathrm{A}$ also includes a third intrachain disulfide 67 bridge, situated between residues 6 and $11 .{ }^{13}$ On the other 68 hand, CS is a biodegradable, biocompatible, and nontoxic 69 polymer obtained from chitin. It is known that chitin is a 70 byproduct that becomes an environmental pollutant derived 71 from activities in fish farms. After a chemical modification, not 72 only is the byproduct used but a new product with added value 73 and new properties is obtained. The presence of $-\mathrm{NH}_{2}$ and $74-\mathrm{OH}$ groups gives to $\mathrm{CS}$ interesting chemical-biological 75 properties. ${ }^{14}$ The literature cites uses and properties for this 76 polysaccharide as varied as enzyme immobilization, antimicro77 bial agent, in the preservation of food, promoter of plant 78 growth, and absorption of metals and dyes, to name a few. ${ }^{15}$

79 As for INS and CS, these two versatile polymers allow the 80 generation of a wide variety of complexes: it has been reported 81 that bioadhesive chitosan-coated cationic nanoliposomes 82 showed improved INS encapsulation and a prolonged oral 83 hypoglycemic effect in diabetic mice. ${ }^{16}$ Furthermore, CS84 lecithin liposomal nanovesicles ${ }^{17}$ have been tested as new 85 carriers for oral delivery of INS, while Sadhasivam et al. have 86 developed patches in order to evaluate the encapsulation 87 efficiency and the release rate for transdermal delivery of 88 INS. $^{18}$

89 Moreover, INS-CS nanoparticle formation was explored by 90 our group. ${ }^{19}$ In this previous contribution a model that would 91 explain the nanocomplex formation between human recombi92 nant INS and a high molecular weight CS was proposed in 93 order to be administrated through alternative ways than the 94 parenteral, for instance oral or pulmonary. ${ }^{20-22}$ However, one 95 of the most interesting features of the nanocomplexes is their 96 potential to trap and release the hormone they contain in a 97 controlled manner. This release will depend, among other 98 factors, on the $\mathrm{pH}$, the temperature, or the ionic strength of the 99 surrounding medium. ${ }^{23}$ The INS and CS concentration 100 conditions that allowed the formation of complexes were 101 established and complexes with defined and reproducible submicronic dimensions were obtained. Complex formation 102 was modulated by $\mathrm{pH}$ as the driving force involved in the 103 process and the main type of chemical interactions between 104 INS and CS were the electrostatic ones, established between 105 specific titratable groups of each biopolymer: amine for CS and 106 carboxylic for INS.

It is well-documented in the literature that the interplay 108 between biocolloidal characteristics as size and charge, and 109 extrinsic macromolecular properties as $\mathrm{pH}$, ionic strength, and 110 thermal energy results in a unique collection of mesoscopic 111 forces for the molecular configuration and functionality in 112 biological systems. ${ }^{24,25}$ Different electrostatic coupling regimes 113 can be observed depending on these physical chemical 114 parameters. This work focus on the system constituted by 115 INS and CS in a weak coupling regime, i.e. where attraction 116 between both biopolymers can be experimentally seen and 117 when they carry electric net charges of the same signal instead 118 of the expected repulsive behavior as predicted by the classical 119 DVLO theory. ${ }^{26}$ This phenomenon can be described by 120 Kirkwood's structure-sensitive electrostatic forces, ${ }^{27}$ where 121 mesoscopic attractive forces between macromolecules arise 122 from fluctuations in titratable amino acid charges due to the 123 induced shifts in the acid-base equilibrium. The attraction is 124 $\mathrm{pH}$-dependent and a result of an intrinsic physical property of 125 the macromolecule, the capacitance (or the protein charge 126 regulation parameter), which is a measure of the macro- 127 molecular net charge fluctuations (defined by the variance of 128 the mean electrical charge) that is the determinant property for 129 the "charge regulation mechanism". ${ }^{28-30}$ The protein 130 capacitance varies with solution $\mathrm{pH}$ as a consequence of the 131 number of amino acid residues that titrate around each $\mathrm{pH}^{25}{ }^{25}$ The attraction between charges alike has been called 133 "complexation on the wrong side" of $\mathrm{p} I$ and reported in the 134 literature for many other systems. ${ }^{31,24,25,30,32} 135$

The objective of this work was to investigate theoretically 136 and to demonstrate via constant $-\mathrm{pH}$ Monte Carlo simulations 137 that the complexation phenomenon (noncovalent linkages) 138 between INS and the cationic polyelectrolyte CS is mainly 139 driven by an electrostatic mechanism. The complexation 140 process was modeled by a constant- $\mathrm{pH}$ scheme as it is well- 141 known that $\mathrm{pH}$ can induce attraction between two titratable 142 objects that results in stronger macromolecular association. 143 The effect of the oligomeric states of INS was also investigated 144 demonstrating that the number of INS molecules affects the 145 complexation phenomena. CS self-association was assumed to 146 be of minor importance to describe the main features of the 147 INS-CS complexation and therefore is not explicitly included 148 in the molecular model. The inclusion of a larger polymer 149 chain or more chains would hamper the CPU time. 150 Experimental results obtained from FTIR spectra and $\zeta-151$ potential determinations allowed us to complement the 152 conclusions.

\section{MATERIALS AND METHODS}

2.1. Materials. $\mathrm{CS}, \mathrm{MW}=300 \mathrm{kDa}$ and deacetylation 154 degree (DD) of 72\%, was kindly donated by the Microbiology 155 Laboratory of Instituto Nacional de Tecnologia Industrial of 156 Mar del Plata, Argentina. CS solutions were prepared with 1\%, 157 $\mathrm{w} / \mathrm{w}$, acetic acid.

Recombinant human INS was supplied by Denver Farma 159 Laboratories, Buenos Aires, Argentina. The exact mass of INS 160 was dissolved in $20 \mathrm{mM} \mathrm{HCl}$ solution, $\mathrm{pH} 2$. 


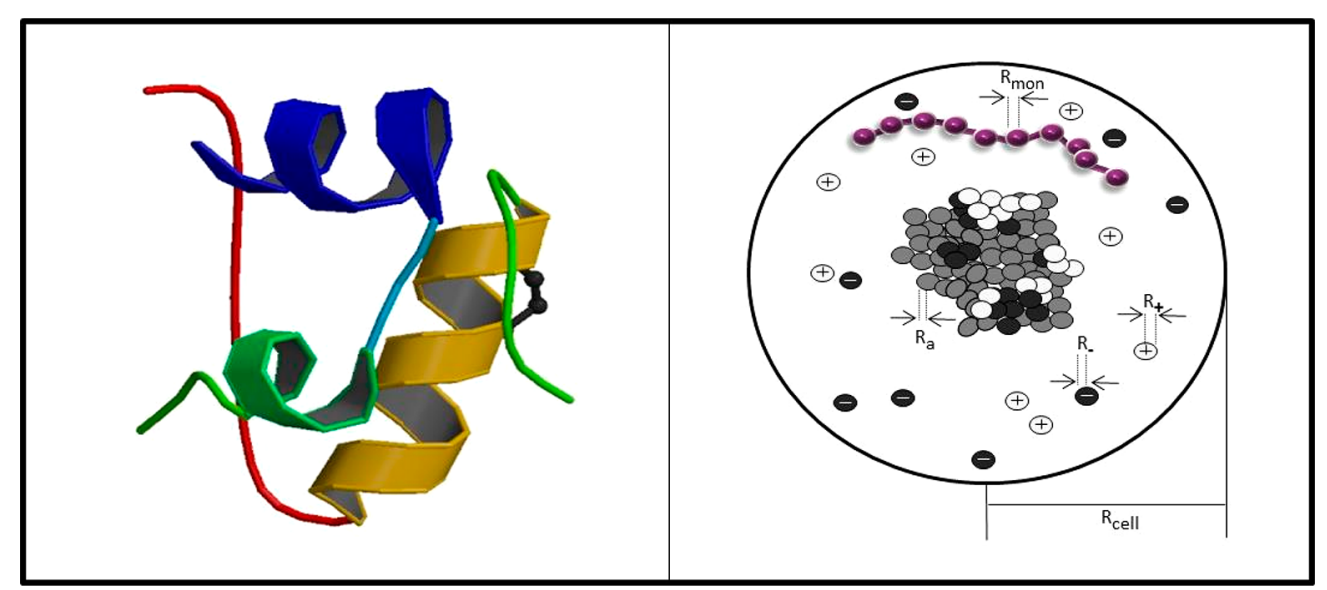

Figure 1. Macromolecular representations. (left) Structure of INS (chain A and B in the monomeric form at $\mathrm{pH}$ 6) as given by the crystallographic coordinates (PDB id 1APH). (right) Schematic representation of the INS-CS model system. INS is given in atomic details in a spherical electroneutral cell (radius $R_{\text {cell }}$ ) and is seen surrounded by counterions and added salt particles. Positive and negatively charged protein atoms (radii $R_{\mathrm{a}}$ ) are represented in white and black, respectively. A flexible polyelectrolyte chain is used to model CS. The size of its monomers is $R_{\text {mon }}$.

162 All other chemicals were of analytical quality. Milli-Q water 163 was always used. CS solutions were kept at $4{ }^{\circ} \mathrm{C}$ for $24 \mathrm{~h}$ to 164 achieve the complete hydration of its molecules before its use. 165 2.2. Preparation of INS-CS complexes. For INS-CS 166 nanoparticle preparation, each double concentrated biopol167 ymer solution was mixed at $\mathrm{pH} 2$, to give the required final 168 concentrations of protein and polysaccharide in the bulk 169 solution. INS solution was poured into CS solution under 170 gently magnetic stirring and the mixed solution $\mathrm{pH}$ was 171 increased up to 6 by careful addition of $\mathrm{Na}(\mathrm{OH}), 4 \mathrm{~N}$, 172 solution, added drop by drop under stirring.

173 2.3. Optical Density Measurements. Each solution was 174 freshly prepared and its optical density (OD) was registered 175 upon time to study the formation of INS-CS complexes, at $176 \mathrm{pH} 2$ and $6^{33,34}$ using a PHERASTAR-FS microplate reader 177 (BMG LabTech, Ortenberg, Germany). Optical density of INS 178 solution was also registered as a control. Data was acquired at $\lambda$ $179=500 \mathrm{~nm}$, away from INS and also CS intensity peaks. ${ }^{35}$ 180 Measurements were all performed at $25{ }^{\circ} \mathrm{C}$; $200 \mu \mathrm{L}$ of each 181 solution was poured into each well with no additional 182 preparation.

183 2.4. $\zeta$-Potential Determinations. Briefly, INS and CS 184 solutions were freshly prepared and their $\zeta$-potential was 185 measured for a $\mathrm{pH}$ range between 2 and 8 . These 186 measurements were carried out using a Zetasizer Nano-Zs 187 particle analyzer (Malvern Instruments, Worcestershire, UK), 188 as described by Silva et al. ${ }^{19}$

189 2.5. Freeze-Drying. INS and INS-CS solutions were 190 lyophilized using a Stokes freeze-dryer operating at $-40{ }^{\circ} \mathrm{C}$ 191 condenser plate temperature and a chamber pressure of less 192 than $100 \mu \mathrm{m} \mathrm{Hg}$. After that they were dried in a vacuum oven 193 at $40{ }^{\circ} \mathrm{C}$ in the presence of $\mathrm{P}_{2} \mathrm{O}_{5}$ to a constant weight. ${ }^{36}$

194 2.6. Infrared Spectroscopy. Fourier transform infrared 195 (FTIR) spectra of lyophilized INS, CS, and INS-CS solutions 196 were recorded on a Nicolet 8700 FT-IR Spectrometer with an 197 ATR modulus. For each spectrum, a 64-scan interferogram was 198 collected with $4 \mathrm{~cm}^{-1}$ resolution at room temperature. The 199 data was processed with OMNIC 7.3 software from Thermo 200 Electron Corporation. All samples were run in triplicate, and 201 the results showed are the average of three measurements.

202 2.7. Theoretical Modeling. The molecular modeling 203 began by considering the monomeric Human INS (chains A and B) in full atomistic details as a rigid body formed by a 204 collection of charged hard spheres like beads. Each bead 205 mimics a protein atom and is placed at the spatial coordinates 206 given by the crystallographic structure available at the Protein 207 Data Bank (PDB id 1APH) (Figure 1 left). This PDB file was $208 \mathrm{fl}$ edited before the calculations. All water molecules and 209 heteroatoms were removed. The assigned radius for the INS 210 beads (atoms) is $2.0 \AA$ as suggested before in refs 37 and 32. 211 Partial charges are assigned to beads depending on the physical 212 chemical nature of the amino acids and as a function of the 213 solution $\mathrm{pH}$. Only specific atoms from titratable chemical 214 groups [ $\alpha$-carboxyl, aspartyl carboxyl, glutamyl carboxyl, 215 imidazole, $\alpha$-amino, thiol (when not involved in SS bridges), 216 phenolic, amino, and guanidyl] can be charged. All other atoms 217 are assumed to be neutral. The protonation state of the 218 ionizable groups can be altered during the simulations. This 219 acid/base equilibrium is controlled by a protein titration 220 scheme that considers any change in the environment 221 including the $\mathrm{pH}$, ionic strength, molecular concentration, 222 presence of other charged objects (e.g., polysaccharide chain), 223 and any variation of the protonation state of other titratable 224 groups. This numerical scheme has been detailed described in 225 previous works. $^{37,32,38}$ Different homo-oligomeric states were 226 investigated. INS dimers, trimers, tetramers, and hexamers 227 were built using the "GalaxyHomomer" server" ${ }^{39}$ with default 228 parameters from the crystallographic structure (PDB id 1APH) 229 in the monomeric form. This was necessary because our 230 simulations did not include the self-aggregation process for 231 INS. Since the focus of the work is on INS-CS complexation, 232 we assumed that the INS homoassociation happened before its 233 interaction with the polyelectrolyte chain. Therefore, an initial 234 structure (dimer, tetramer, etc.) was chosen to be studied and 235 calculations were repeated for all oligomeric states as there 236 were done for the monomeric case.

For all oligomeric states, the protein INS as a monomer, 238 dimer, trimer, tetramer, or hexamer was kept rigid, static, and 239 in the same molecular configuration at the center of an 240 electroneutral spherical cell with radius $R_{\text {cell }}$ (Figure 1 right) 241 determined by the protein concentration. ${ }^{37}$ This corresponds 242 to the so-called "cell model". 40

Polyelectrolytes, such as CS, can be modeled as a flexible 244 chain of charged monomers (beads) linked together with 245 
246 harmonic springs. Following previous works, ${ }^{37,32}$ we used a 247 polycation chain with 21 beads with a radius $\left(R_{\text {mon }}\right)$ of $2 \AA$. 248 This is a reasonable number of monomers to describe the main 249 physical interactions keeping a good balance between the real 250 experimental conditions with the efficiency of the computa251 tional sampling. A larger chain would enhance the electrostatic 252 interactions. At the same time, it would increase the CPU time. 253 A uniform size of all beads was necessary to mimic the 254 monomeric units of CS that also have the same size. Such 255 chain is flexible and can freely move inside the cell, interacting 256 with the protein atoms, counterions and added monovalent 257 ions. Complementary simulations were also carried out with a 258 polyanion in order to explore CS in both acid and basic 259 regimes. Only one chain was included in the simulation cell. 260 For the sake of simplicity, we have assumed that the charges of 261 the monomers were fixed and fully protonated (a high degree 262 of deacetylation) for all simulated $\mathrm{pH}$ conditions. This implies 263 that the observed bimolecular interactions for solution $\mathrm{pH} \gg$ 2646.5 (the $\mathrm{p} K_{\mathrm{a}}$ of the amino group in CS is $\sim 6.5$ ) tend to be 265 overestimated. This should have a minor impact in our study 266 since we are working on $\mathrm{pH}<8$, and most of the practical 267 applications of CS are at the acid regime. ${ }^{41}$

268 Added salt and counterions were modeled by the usual 269 restricted primitive model. ${ }^{42}$ Each mobile ion $k$ (added salt and 270 counterions), with a $q_{k}$ charge is considered as a charged hard 271 sphere of radius $R_{\mathrm{a}}$ equals to $2.0 \AA$, while the solvent is 272 effectively considered as a continuous dielectric medium 273 without an explicit molecular structure and characterized by 274 its relative static dielectric permittivity $\varepsilon_{s}$.

275 The combination of the cell model with the primitive one, 276 the titration scheme and the modeling procedure for the 277 polyelectrolyte chain defines the constant-pH coarse grained 278 (CG) model, previously described by Barroso da Silva and 279 coauthors $^{37,32,43,44,25}$ aiming to explore the protein complex280 ation phenomena with the focus on electrostatic interactions 281 and invoking a minimum set of parameters. The reader is 282 referred to these cited papers ${ }^{37,32,43,44,25}$ for more details about 283 the model and the chosen parameters.

284 The interaction potential energy between any two particles $i$ 285 and $j\left[U^{\text {ele }}\left(r_{i j}\right)\right]$ is given the combination of the Coulombic 286 interaction with the hard-sphere model (eq 1 ):

287

$$
U^{\text {ele }}\left(r_{i}\right)= \begin{cases}\frac{q_{i} q_{j} e^{2}}{4 \pi \varepsilon_{0} \varepsilon_{s} r_{i j}}, & r_{i j} \leq R_{i}+R_{j} \\ \infty, & \text { otherwise }\end{cases}
$$

288 where $\varepsilon_{0}$ is the vacuum permittivity, $q_{i}$ and $q_{j}$ are the particles 289 charge $i$ (radius $R_{i}$ ) and $j$ (radius $R_{j}$ ), respectively, and $r_{i j}$ is 290 their separation distance. This potential of interaction without 291 a van der Waals attractive term guarantees that attraction is 292 only possible via the electrostatic interactions.

293 The polyelectrolyte (polycation or polyanion) monomer294 monomer interaction $\left(\beta u^{\text {bond }}\right)$ was modeled by (eq 2 )

295

$$
\beta u^{\text {bond }}=\frac{l_{\mathrm{b}}}{2 r_{\text {min }}^{3}} \sum_{i=1}^{N_{\text {mon }}-1}\left(r_{i, i+1}\right)^{2}
$$

296 where $l_{\mathrm{b}}$ is the Bjerrum length, $r_{j i+1}$ is the separation between 297 the consecutive monomers $i$ and $i+1, r_{\min }$ is the separation 298 corresponding to the energy minimum for a dimer. As in 299 previous studies, we have assumed $r_{\min }$ equals to $4 \AA$ which results in an average monomer-monomer separation of 300 approximately $7.4 \AA$.

All the mobile particles (polyelectrolyte beads, counterions, 302 and added salt particles) are kept inside the simulation cell via 303 an external potential $\left[U^{\text {ext }}\left(r_{i}\right)\right]$ (eq 3$)$ :

304

$$
U^{\text {ext }}\left(r_{i}\right)= \begin{cases}0, & r_{i} \leq R_{\text {cell }} \\ \infty, & \text { otherwise }\end{cases}
$$

The total energy of the system for a given configuration 306 $\left[U\left(\left\{\boldsymbol{r}_{\boldsymbol{k}}\right\}\right)\right]$ is given by the combination of eqs $1-3$ :

$$
U\left(\left\{\boldsymbol{r}_{\boldsymbol{k}}\right\}\right)=\sum_{i=1}^{N_{\mathrm{c}}+N_{\mathrm{s}}} U^{\mathrm{ext}}\left(r_{i}\right)+\frac{1}{2} \sum_{i=1}^{N} \sum_{j=1}^{N} U^{\mathrm{ele}}\left(r_{i j}\right)+\beta u^{\text {bond }}
$$

where $N_{\mathrm{c}}$ is the number of counterions, $N_{\mathrm{s}}$ is the number of 309 added salt particles, and $N=N_{\mathrm{c}}+N_{\mathrm{s}}+N_{\mathrm{P}}+N_{\text {mon }}$, which also 310 includes the total number of charged protein atoms $\left(N_{\mathrm{P}}\right)$ and 311 the number of polyelectrolyte monomers $\left(N_{\text {mon }}\right)$. Note that 312 the $N_{c}$ is a different number when the polyelectrolyte is a 313 polycation ( $\mathrm{CS}$ for $\mathrm{pH}<6.5$ ) or a polyanion ( $\mathrm{CS}$ for $\mathrm{pH} \geq 314$ $6.5)$.

2.8. Simulation Details. The computer simulations were 316 performed in a semigrand-canonical ensemble using the 317 standard Metropolis Monte Carlo (MC) algorithm ${ }^{45}$ with 318 random translational displacements of mobile species 319 (counterions, added salt particles, and polyelectrolyte mono- 320 mers) within the electroneutral simulation cell and the titration 321 movements. ${ }^{37}$ A proton reservoir was set up within the cell to 322 define a constant $\mathrm{pH}$ for the system. The solved constant-pH 323 $(\mathrm{CpH}) \mathrm{CG}$ model keeps constant the number of particles 324 while the individual charges can vary during the simulation due 325 to the protonation/deprotonation process. ${ }^{32} 326$

For each simulation, at least $10^{9} \mathrm{MC}$ steps were necessary 327 for the system equilibration. They were followed by $10^{10}$ MC 328 steps for production that were used for the measurements and 329 analyses. $R_{\text {cell }}$ was assumed to be equal to $150 \AA$ which 330 corresponds to a protein concentration of $58.7 \mathrm{mM}$. Salt 331 concentration $(\mathrm{NaCl})$ was fixed at $1.2 \mathrm{mM}$, while the solution 332 $\mathrm{pH}$ was varied from 4 to 8 . Temperature was $298 \mathrm{~K}$, and 333 therefore, $\varepsilon_{\mathrm{s}}$ was 78.7 in all simulation runs. At least three 334 simulation replicas were carried out to confirm the 335 convergence.

The main physical chemical quantity necessary for the 337 present discussion is the free energy of interactions. Never- 338 theless, simulations with flexible chains in a solvent are well- 339 known to be a special difficult case for free energy calculation 340 due to the large energy barriers. ${ }^{46}$ This problem can be solved 341 by means of the penalty function protocol that we proposed in 342 a previous work. ${ }^{37}$ Alternatively, as it was recently demon- 343 strated, ${ }^{32,44}$ the sampling can also be sped up and the CPU 344 time saved by means of the calculation and analysis of the 345 product between the radial distribution function $[g(r)]$ and the 346 square of the separation distance $\left(r^{2}\right)$. Therefore, we followed 347 this second protocol here to reduce the CPU time and be able 348 to study larger oligomeric states. During the production runs, 349 $g(r)$ was computed for the polymer beads-protein center 350 based on histograms with $1 \AA$ size. After the simulation, $g(r) 351$ were multiplied by $r^{2}$ and analyzed as done in refs 44 and 47.352 


\section{RESULTS}

353 3.1. $\mathrm{pH}$ Determinations. In order to experimentally 354 determine the optimal $\mathrm{pH}$ that would maximize electrostatic 355 interactions between hexameric INS and CS, $\zeta$ potential 356 determinations were carried out. Such $\zeta$ potential values $(\mathrm{mV})$ 357 can be seen in Table 1 . It is important to notice that initial $\mathrm{pH}$

Table 1. $\zeta$-Potential Values $(\mathrm{mV})$ vs Solution $\mathrm{pH}$ for INS and CS Solutions ${ }^{a}$

\begin{tabular}{crr}
$\mathrm{pH}$ & \multicolumn{1}{c}{ INS } & \multicolumn{1}{c}{ CS } \\
3 & $20.6 \pm 2.1$ & $5.8 \pm 2.8$ \\
4 & $17.4 \pm 1.3$ & $6.8 \pm 0.7$ \\
$\mathbf{5}$ & $-\mathbf{9 . 5} \pm \mathbf{0 . 7}$ & $\mathbf{6 . 5} \pm \mathbf{0 . 5}$ \\
$\mathbf{6}$ & $-\mathbf{3 2 . 4} \pm \mathbf{2 . 2}$ & $\mathbf{5 . 5} \pm \mathbf{0 . 7}$ \\
8 & $-39.1 \pm 3.3$ & $-17.9 \pm 0.8$ \\
9 & $-44.6 \pm 1.9$ & $-20.5 \pm 1.6$
\end{tabular}

${ }^{a}$ Ionic strength $I=0.15 \mathrm{M}$. INS $0.2 \% \mathrm{w} / \mathrm{v}$, CS $1 \times 10^{-3} \% \mathrm{w} / \mathrm{v}$, at 25 ${ }^{\circ} \mathrm{C}$. The number of INS units depends on the considered $\mathrm{pH}$. Mean \pm SD, $n=3$.

358 conditions for INS correspond to the dimeric state for the 359 protein, ${ }^{48}$ but with increasing $\mathrm{pH}$, the number of INS units 360 also increases and corresponds to the hexameric conformation 361 of the protein when the system reaches $\mathrm{pH} 6$.

362 For acidic pHs, lower than 5, both species (INS and CS) 363 present positive net charges, therefore, attractiveness forces via 364 electrostatic Coulombic interactions would be ruled out into 365 the DVLO framework, ${ }^{26}$ the interactions between like charged 366 macroparticles are always repulsive due to the repulsive 367 Coulombic contribution. In a similar manner, for alkaline $368 \mathrm{pHs}$ greater than $8, \zeta$-potential values are negative for both 369 INS and CS, and electrostatic Coulombic repulsion is to be 370 expected. In a pure Coulombic context, based on the $\zeta$ 371 potentials, the INS-CS complexation could only be observed 372 for the solution $\mathrm{pH}$ window between 5 and 6 (see the 373 highlighted data in Table 1).

374 On the other hand, the experimentally determined $\zeta$ 375 potential corresponding to mixed systems for $4.5<\mathrm{pH}<6$, 376 hints that the probability of electrostatic interactions decreases, 377 because the superficial charge of both macromolecules presents 378 the same sign for this $\mathrm{pH}$ solution range. ${ }^{19}$ A turbidity 379 increases in mixed solutions, by naked-eye observation, also 380 would indicate INS-CS interactions.

381 Such a phenomenon was not observed for single INS and $382 \mathrm{CS}$ solutions at this $\mathrm{pH}$ range. It is important to note that 383 interactions of different physical natures than electrostatic, 384 such as hydrophobic, van der Waals forces, etc., could be 385 taking an important role, even though local electrostatic 386 interactions between charged groups could not be completely 387 discarded.

388 The INS molecule contains many ionizable groups, due to 6 389 amino acid residues capable of attaining a positive charge and 39010 amino acid residues capable of attaining a negative charge. 391 The net charge of the INS molecule as a function of $\mathrm{pH}$ 392 solution calculated on the basis of amino acids intrinsic $\mathrm{p} K_{\mathrm{a}}$ 393 values was found by Tanford and Epstein. ${ }^{49}$ The net charge is 394 zero at $\mathrm{pH} 5.5$, in good agreement with the electrophoretically 395 determined isoelectric $\mathrm{pH}$ of 5.3-5.4. ${ }^{50}$ These results are also 396 in accordance with those obtained by a combination of 397 modern techniques, as presented in the Open Chemistry Data 398 Base, on the Pub Chem Web site (National Center for
Biotechnology Information. PubChem Compound Database; 399 CID $=16129672)$. $^{73}$

Our results showed that under the following experimental 401 conditions, $0.2 \% \mathrm{w} / \mathrm{v}, I=0.15 \mathrm{M}, 25^{\circ} \mathrm{C}$, INS presented a net 402 zero charge at $\mathrm{pH}$ 5.0, as obtained by experimental $\zeta$ potential 403 determination. ${ }^{19}$ The discrepancy among the experimental and 404 reported data, could obey to the different methodological 405 techniques applied and other physical chemical parameters as 406 the ionic strength, protein concentration or even a different 407 protein genetic variant. For instance the pioneering work of 408 Wintersteiner and Abramson in $1933^{51}$ found a $\mathrm{pI}$ value for 409 INS of 5.4 derived from turbidity measurements, which was in 410 accordance with the result of the electrophoretic results from 411 adsorbed or amorphous INS presented in the same 412 contribution. The authors also found a $\mathrm{pI}$ value of 5.55 to 413 5.60 derived from the solubility based on measurements; which 414 was considered less robust and susceptible to be discarded 415 because the method of determination. Righetti and Caravaggio 416 (1976) reported $\mathrm{pIs}$ for bovine and porcine INS, via isoelectric 417 focusing, with values of 5.72 and 6 , respectively. ${ }^{52}$ As will be 418 commented to a greater extent below, the theoretical predicted 419 $\mathrm{pI}$ for INS obtained by the present constant-pH MC 420 simulations is also within the interval 5.4-5.6 depending on 421 the oligomeric state.

422

Taking into account the experimentally determined INS pI 423 and considering that the $\mathrm{CS} \mathrm{pK}_{\mathrm{a}}$ is reported to be $6.5,{ }^{41}$ a 424 solution $\mathrm{pH}$ of 6 was selected for the INS-CS nanocomplex 425 generation. At this solution $\mathrm{pH}$, the macromolecular attraction 426 is expected to be maximum due to the net charges of INS and 427 CS in a Coulombic framework.

428

3.2. Optical Density Measurements. INS aggregation 429 kinetics were monitored by registering changes in optical 430 density (OD) of INS-CS solutions over time, for solution $\mathrm{pH} 431$ 6 and ionic strength of $0.15 \mathrm{M}$. Turbidimetric studies applied 432 to protein-polysaccharide systems are based on the fact that 433 turbidity/OD is proportional to both size and concentration of 434 the particles formed. ${ }^{53}$ The experimental data was then fitted 435 using the model proposed by Stirpe et al., ${ }^{54}$ a non-linear square 436 curve-fitting to the following stretched exponential function 437 (eq 5):

$$
\mathrm{OD}(t)=\mathrm{OD}+\gamma \exp (-\alpha \mathrm{t})^{\beta}
$$

where $\mathbf{O D}, \mathbf{O D}_{0}$, and $\gamma$ are the observed optical density value 440 at time $t$, the final optical density value, and the preexponential 441 factor defined as optical density amplitude, respectively, 442 whereas $\boldsymbol{\alpha}$ and $\boldsymbol{\beta}$ are the rate and the order of spontaneous 443 aggregate formation. ${ }^{54}$ Fitting data and the mathematical 444 model applied corresponding to the kinetic parameters can be 445 seen in Supporting Information. Values of $\boldsymbol{\alpha}$ and $\boldsymbol{\beta}$ derived 446 from the fitting procedure in single INS and INS-CS 447 complexes are reported in Table 2.

$448 \mathrm{t} 2$

As it can be seen in Table 2, the INS-CS rate of protein 449 aggregation almost triplicates the corresponding rate of single 450

Table 2. Kinetic Parameters That Describe the Protein Aggregation Process, from Experimental Data Fitting ${ }^{a}$

$\begin{array}{lcc}\text { sample } & \alpha(1 / \mathrm{min}) & \beta \\ \text { I } & 0.29 \pm 0.01 & 2.2 \pm 0.9 \\ \text { INS-CS } & 0.76 \pm 0.16 & 2.1 \pm 0.8\end{array}$

${ }^{a}$ Mean \pm SD, $n=3$. Ionic strength $I=0.15 \mathrm{M}$, INS $0.2 \% \mathrm{w} / \mathrm{v}$, CS $1 \times$ $10^{-4} \% \mathrm{w} / \mathrm{v}$. 
451 INS. For single INS, the absence of solutes determines low 452 rates of aggregation, behavior that notoriously changes with the 453 presence of CS chains forming a dilute solution regime. ${ }^{55}$

454 The order of protein aggregates formation was similar for 455 INS and INS-CS solutions, which could indicate that for this 456 CS concentration, interactions between the protein and the 457 polysaccharide chains were favored, i.e., both species were 458 easily accessible due to, in part, the bridging flocculation 459 phenomenon. Bridging-flocculation phenomenon is an analogy 460 to the concept used in emulsion science. Briefly, the long 461 polysaccharide chains are able to bind more than one protein 462 aggregate, forming protein molecular clusters. Thus, each 463 cluster is formed by charge neutralization and bridging effects. 464 This effect is most pronounced at lower polysaccharide 465 concentrations where protein aggregates surfaces are only 466 partially covered. Under this phenomenon, long polysaccharide 467 chains can bind protein aggregates and form molecular clusters 468 due to charge neutralization and bridging effects; therefore not 469 many intermediate steps were needed toward complex470 ation. ${ }^{56,57}$

$471 \quad 3.3$. FT-IR Analysis. The interactions between INS and CS 472 during the complexation process can be traced using infrared 473 spectroscopy by comparing single INS and CS spectra with the 474 spectrum obtained for the mixture (Figure 2). As can be seen, 475 INS exhibits typical peaks around 1650 and $1540 \mathrm{~cm}^{-1}$, that 476 correspond to $\mathrm{C}=\mathrm{O}$ stretching and $\mathrm{C}-\mathrm{N}$ and $\mathrm{N}-\mathrm{H}$ 477 interactions, respectively. ${ }^{58}$

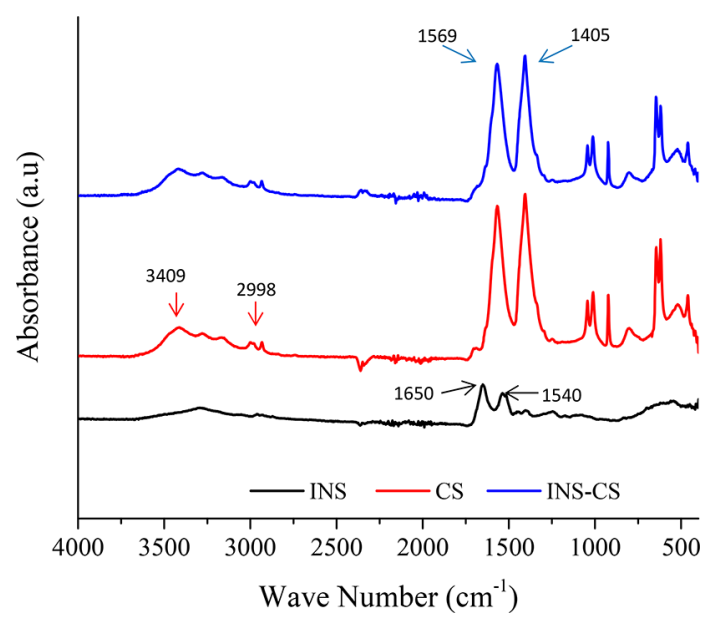

Figure 2. Infrared spectra of lyophilized INS, CS, and INS-CS complexes in the field $4000-500 \mathrm{~cm}^{-1}$, at $25{ }^{\circ} \mathrm{C}$ and $I=0.15 \mathrm{M}$. Solutions were prepared at $\mathrm{pH} 6$ prior to lyophilization.

478 On the other hand, the CS spectrum shows peaks around 4793409 and $3283 \mathrm{~cm}^{-1}$ (O-H and $\mathrm{N}-\mathrm{H}$ stretching), $2998 \mathrm{~cm}^{-1}$ 480 (C-H stretch vibrations.), $1567 \mathrm{~cm}^{-1}$ ( $\mathrm{C}=\mathrm{O}$ tension in 481 amide $\mathrm{I}), 1404 \mathrm{~cm}^{-1}(\mathrm{C}-\mathrm{H}$ and $\mathrm{O}-\mathrm{H}$ deformation in amide $482 \mathrm{II}), 1013 \mathrm{~cm}^{-1}$ (C-O and $\mathrm{C}-\mathrm{H}$ tension), and $808 \mathrm{~cm}^{-1}$ 483 associated with the glucosamine units. ${ }^{59-62}$

484 In INS-CS complexes spectrum, single INS mean peaks 485 ( 1650 and $1540 \mathrm{~cm}^{-1}$ ) are masked. The characteristic 486 absorption bands for the complexes appeared at 1569 and $4871405 \mathrm{~cm}^{-1}$. The first one could be assigned to a combination of 488 signals from CS amide I and INS amide II. The second peak 489 corresponds to $\mathrm{CS}$; the small displacement from single CS 490 spectrum could be associated with the interaction between the 491 polymer and the protein at the level of amide II. No new peaks emergence was observed for the INS-CS complexes spectrum, 492 which is in coincidence with the results obtained by Prusty and 493 Sahu. ${ }^{63}$ These observations suggest that some weaker physical 494 interactions between INS and the polymer were formed during 495 complexes formation, at least under these conditions of 496 macromolecules concentration, $\mathrm{pH}$, ionic strength, temper- 497 ature, and solvent characteristics.

The findings presented in this contribution, regarding the 499 effect upon complexation visualized through INS-CS FTIR 500 spectra, are in line with those previously reported by 501 Mukhopadhyay et al. and Sarmento et al. ${ }^{58,64} 502$

3.4. Numerical Simulations. In a pure DVLO approach 503 the main physical driving force for the protein-polyelectrolyte 504 complex formation is the Coulombic attraction, for oppositely 505 charged particle systems. Conversely, for macromolecular 506 species with like charges, the interaction would be always 507 repulsive in this context. For macromolecules, however, the 508 formation of soluble complexes can occur even when the 509 protein and the polyelectrolyte present net charges with the 510 same sign. ${ }^{37,32,44}$ This has also been well reported in the 511 literature in several experimental papers, for poly diallyl- 512 dimethylammonium chloride (PDADMAC) and $\beta$-lactoglobu- 513 $\mathrm{lin}^{65}$ for a cationic gold nanoparticle coupled to $3,6,9,12-514$ tetraoxatricosan-1-aminium, 23-mercapto- $N, N, N$-trimethy 515 (TTMA) and $\mathrm{BSA}^{66}$ and several dendrimers and lysozyme, ${ }^{67} 516$ just to cite a few examples.

As a result of a perturbation in the acid-base equilibrium, 518 several changes can be induced in the distributions of charged 519 groups, leading to the charge regulation mechanism enhance- 520 ment. ${ }^{44}$ These proton fluctuations evolve into an attractive 521 mesoscopic electrostatic force, which in certain circumstances 522 can overcome electrostatic Coulombic repulsion and allow 523 complexes formation in the so-called "wrong side of pI" ${ }^{68}$ This 524 charge regulation phenomenon becomes particularly relevant 525 under conditions, such as low ionic strength and $\mathrm{pH}$ close to 526 the protein's $\mathrm{pI} .^{37,32}$ It is at the $\mathrm{p} I$ that the repulsive Coulombic 527 electrostatic interactions vanish, and the system should be 528 dominated by the attractive interactions. However, depending 529 on the magnitude of the main molecular characteristics (net 530 charges and their charge regulation parameters) and the salt 531 concentration, the attraction can be seen even further from the 532 $\mathrm{p} I$.

In order to comprehend the molecular mechanisms behind 534 the INS-CS complexation mechanisms, simulations were 535 performed at different $\mathrm{pHs}$ (the same $\mathrm{pH}$ range considered for 536 experimental studies), while the number of INSs units was also 537 varying. Along with a single chitosan chain, monomeric, 538 dimeric, tetrameric, and hexameric INSs were considered [CS 539 + nINS]. Thanks to these sets of simulations with different INS 540 oligomeric states, it is possible to obtain the more adequate 541 experimental conditions in terms of the solution $\mathrm{pH}$ and the 542 number of INS units, which would favor complex formation. In 543 this way a contrast between theoretical outcomes with the 544 behavior given by the experimental techniques could be 545 established. Additional $\mathrm{CpH} \mathrm{MC}$ simulations runs were also 546 performed with INS in the presence of an electrolyte solution 547 as well as in the absence of CS. The purpose of this approach 548 was to theoretically predict its isolated $\mathrm{pI}$ as a function of the 549 oligomeric state. It was found that under these conditions, $\mathrm{pI} 550$ varies between 5.4 and 5.6. Monomers, dimers, and trimers 551 have a $\mathrm{pI}$ equal to 5.4 while, for the tetramer, the $\mathrm{p} I$ increases 552 to 5.5 and, for the hexamer, it is equal to 5.6 . 

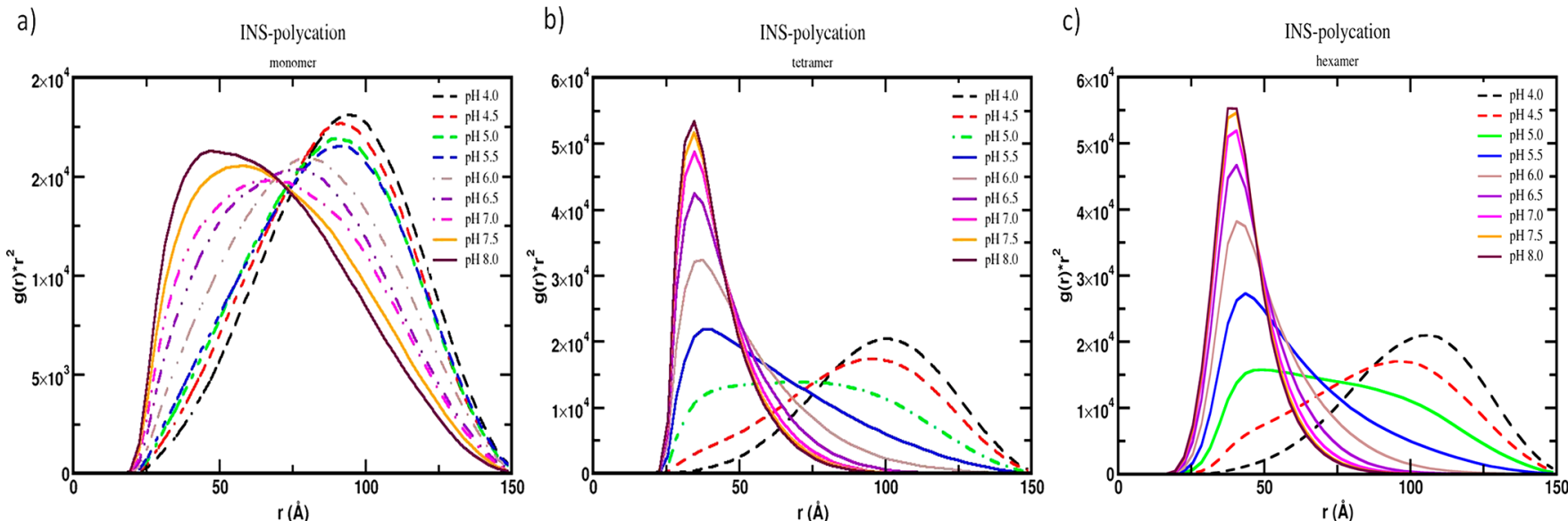

Figure 3. Computed free energy derivatives as a function of the separation distance between the protein center and the polyelectrolyte beads $[g(r)$. $r^{2}$ ], for different numbers of INS units: (a) monomer; (b) tetramer; (c) hexamer. Different lines represent each complexation regime: solid (Coulombic attraction), dashed and dotted (attraction due the charge regulation mechanism), and dashed (Coulombic repulsion).
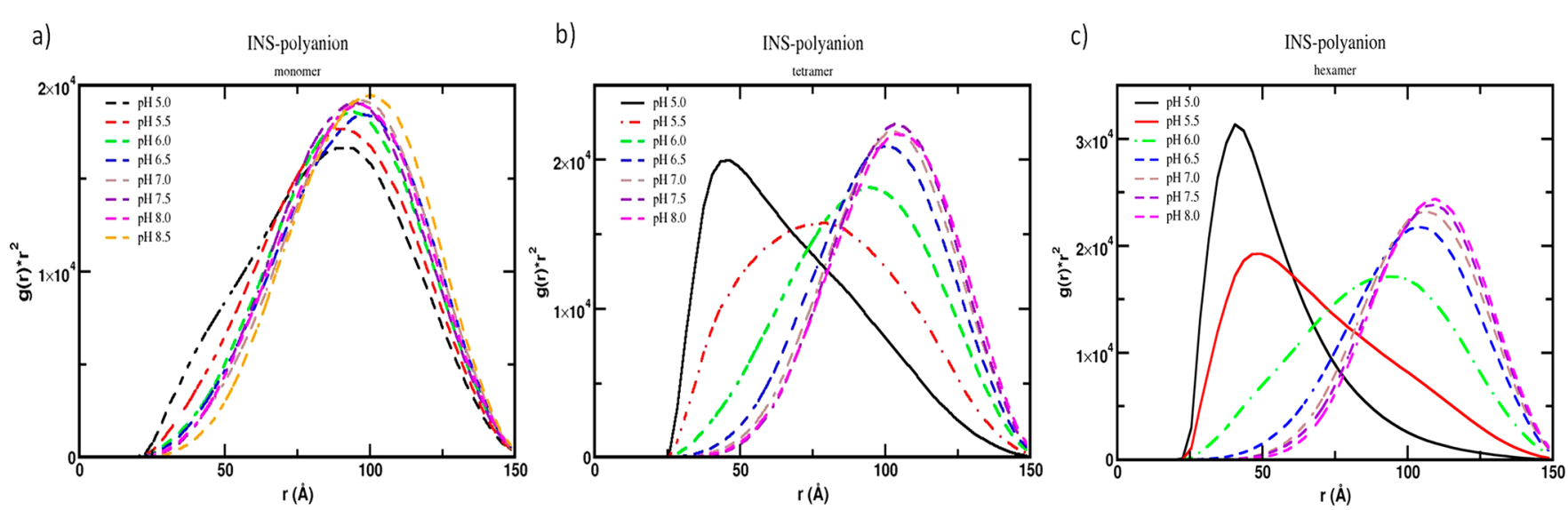

Figure 4. Computed free energy derivatives as a function of the separation distance between the protein center and the polyelectrolyte beads $[g(r)$. $r^{2}$ ], for different numbers of INS units: (a) monomer; (b) tetramer; (c) hexamer. Different lines represent each regime: solid (Coulombic attraction), dashed and dotted (attraction due the charge regulation mechanism), and dashed (Coulombic repulsion).

554 These sets of simulations indicate that the interaction 555 between INS and CS can follow the most common view of 556 Coulombic interactions for some solutions $\mathrm{pH}$ values, where 557 the complexation process requires for the macromolecules to 558 have net charges with opposite sign. Besides these cases, 559 complexation can also be observed for like-charged systems, as 560 pointed out before. These cases with the complexation on the 561 wrong side of $\mathrm{p} I$ are associated with the charge regulation 562 mechanism. ${ }^{37,32,44}$ For all the $g(r) \cdot r^{2}$ plots (see Figures 3-6), 563 the highest the peak at the short separation distance regime, 564 the stronger the attraction becomes. In a similar way, a high 565 peak at a large distance indicates a significant repulsion. As 566 regarding the lines, a solid one indicates complexation, a 567 dotted-dash line suggests the complexation due to the charge 568 regulation mechanism, and a dashed one represents a 569 dominant repulsion mechanism in which no complexation 570 process can be observed.

571 Before introducing the specific results from the different sets 572 of simulations, it is relevant to highlight some experimental 573 considerations from $\zeta$ potential measurements. This is also 574 confirmed by the theoretical predicted titration data: for $\mathrm{pH}<$ 575 5.5, INS and CS present positive superficial charges, while for $576 \mathrm{pH}>6.5$ both charges are negative. The electrostatic 577 Coulombic interaction $\mathrm{pH}$ has been established for $5.5<\mathrm{pH}$
$<6.5$. Any attraction between these macromolecules observed 578 for $\mathrm{pH}>6.5$, when both are negatively charged, would not 579 have a Coulombic origin and could not be explained by the 580 DVLO theory.

3.4.1. Polycation + nINS. Polycation +1 INS. This case 582 corresponds to the system with a CS chain positively charged. 583 In Figure 3, we present an estimative of the interaction free 584 energy in terms of its derivative expressed by the product $g(r) \cdot 585$ $r^{2}$. For the monomeric state of INS, a complexation INS-CS 586 with low affinity can be spotted for alkaline pHs from 7.5 to 8,587 while a wider region where weaker attractions occurs can be 588 seen for $6<\mathrm{pH}<7$. The two macromolecules are not firmly 589 connected in these cases. The weaker attraction is probably 590 due to the charge regulation mechanism that tries to overcome 591 the Coulombic repulsion, not being strong enough to make it 592 concrete. For more acidic $\mathrm{pHs}(\mathrm{pH} \leq 5.5)$, a repulsive 593 interaction was obtained (see Figure 3a) following the classical 594 Coulombic view where two positively charged macromolecules 595 should repeal each other.

596

Polycation + 4INS. In Figure 3b, the effect of monomer 597 number of INS units (aggregates) can be observed in terms of 598 interaction. The change in the oligomeric state from the 599 monomer to the tetramer results in stronger attraction in 600 comparison with the monomeric case, previously exhibited in 601 


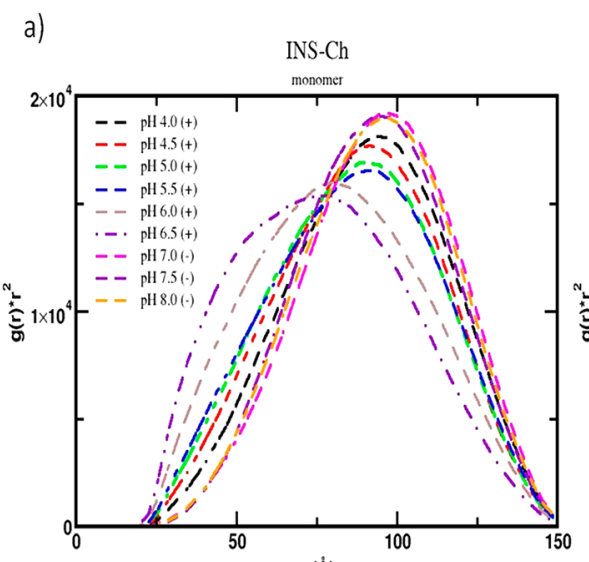

$r(\dot{A})$

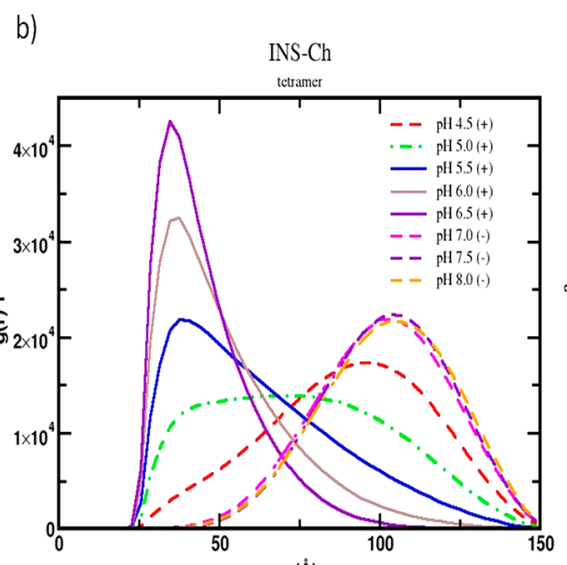

$\mathbf{r}(\AA)$

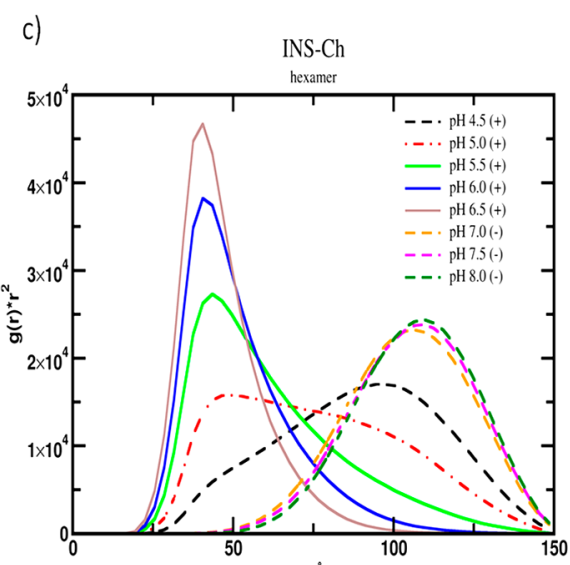

$r(\AA)$

Figure 5. Computed free energy derivatives as a function of the separation distance between the protein center and the polyelectrolyte beads $[\boldsymbol{g}(\boldsymbol{r})$ $r^{2}$ ], for different numbers of INS units: (a) monomer; (b) tetramer; (c) hexamer. Different lines represent each regime: solid (Coulombic attraction), dashed and dotted (attraction due the charge regulation mechanism), and dashed (Coulombic repulsion).

602 Figure 3a. For the tetramer, complexation occurs for a $\mathrm{pH}$ 603 range from 5.5 to 8 , increasing its affinity as the solution $\mathrm{pH}$ 604 value does (Figure $3 \mathrm{~b}$ ). $4 \mathrm{INS}$ (tetramer) is neutral at $\mathrm{pH} 5.5$ 605 which indicates that any attraction cannot be attributed to 606 Coulombic interactions. The highest attraction for the 4INS 607 case was detected at $\mathrm{pH} 8$. On the other hand, $\mathrm{pH} 5$ starts to 608 be a condition where the attraction becomes weaker, and the 609 interaction between the two macromolecules is weaker. The 610 charge regulation mechanism manifests its effect but is not able 611 to overcome the repulsive Coulombic interaction at $\mathrm{pH} 5$. 612 Under these conditions, no clear peak can be observed; hence, 613 this case could be associated with a stage or phase prior to a 614 real complexation, which could be associated with incipient 615 complexation.

616 Polycation + 6INS. For INS on its hexameric form (Figure $6173 \mathrm{c}$ ), the complexation process continued the trend exhibited by 618 4INS (Figure 3b), with the exception that there was no evident 619 charge regulation mechanism and the complexation directly 620 starts at $\mathrm{pH} 5$ and carried on until $\mathrm{pH} \mathrm{8,} \mathrm{where} \mathrm{it} \mathrm{showed} \mathrm{a}$ 621 sharp intensity peak. For this set of simulations, an increase of 622 INS units seemed to play a key role on the complexation 623 process and in the characteristics of the complexes formed.

624 3.4.2. Polyanion + nINS. Polyanion + 1INS. We now turn 625 to the case where the polyelectrolyte should mimic a CS 626 negatively charged. An INS monomer and a polyanionic chain 627 produced no complexation at all studied $\mathrm{pH}$ range, from 5 to 628 8.5, as it can be seen in Figure 4a. All the observed peaks 629 presented separation distances of higher magnitude, which is 630 characteristic of a repulsive interaction among the two 631 macromolecules. It can be inferred from both experimental 632 data and the predicted theoretical titration behavior of INS 633 that, for a $\mathrm{pH}>6.5$, (i.e., CS's $\mathrm{pK}_{\mathrm{a}}$ ), INS and CS have the same 634 negative sign being both species highly soluble. Therefore, 635 electrostatic Coulombic interactions would not be favored for 636 the complexation process to occur by this driving force. ${ }^{25}$

637 Polianion + 4INS. When the number of INS units increases 638 (from 1INS to 4INS), the attraction increases again. 639 Complexation occurred at $\mathrm{pH} \mathrm{5}$, as can be observed in Figure $6404 \mathrm{~b}$, while at $\mathrm{pH} 5.5$ the trend seems to overcome the 641 Coulombic repulsion with charge regulation mechanism. This 642 result indicates that even when the Coulombic electrostatic 643 interactions seem to be dominant in the system, the 644 complexation process carries on due to the increase in the number of INS units. This particle association occurs for $\mathrm{pHs} 645$ in which both species have the same sign in terms of their net 646 charges, or for pHs closer to the protein's pIs. This incipient 647 interaction may arise from the fact that many of INS amino 648 acids have isoelectric points between 5 and 6. For these 649 interaction $\mathrm{pHs}$, some precipitation can be experimentally 650 observed at macroscopic scale, which is a signal of insoluble 651 complex formation.

Polianion + 6INS. Incipient complexation was detected at 653 $\mathrm{pH}$ 6. The complexation process due to Coulombic attraction 654 occurs at $\mathrm{pH} 5$ and 5.5, thus maintaining the tendency for 655 4INS and exhibiting complexation "on the wrong side of $\mathrm{pI}$ ". 656

3.4.3. Chitosan + nINS. In Figure 5, we combined the data $657 \mathrm{fs}$ for the polycation and polyanion mimicking CS at both acid 658 $(+)$ and basic regimes $(-)$. From this data, it can be concluded 659 that complexation process is not expected for INS-CS when 660 INS is in its monomeric form (Figure 5a). For both tetrameric 661 and hexameric forms of INS (Figure 5b, c), incipient 662 complexation due to charge regulation mechanism only took 663 place at $\mathrm{pH} 5$ and complexation range was observed to be $5.5<664$ $\mathrm{pH}<6.5$.

665

When the number of INS units increases in the simulation 666 process, the solution $\mathrm{pH}$ at which the complexation can occur 667 shifts toward acidic conditions. For example, while considering 668 3INS (not shown), this mechanism arises for $5.5<\mathrm{pH}<6.5 .669$ Under these conditions, the highest intensity complexation 670 occurs for pHs 6 and 6.5. Such behavior resembles the 671 experimental conditions: for $\mathrm{pH} \mathrm{5}$, where complexation and 672 precipitation started, and at $\mathrm{pH} 6$ where electrostatic 673 interactions are maximized. While considering a protein 674 aggregate of 4INS $(\mathrm{pI}=5.5)$, complexation occurred for a 675 range of $\mathrm{pHs}, 5.5<\mathrm{pH}<6.5$, with the highest intensity 676 peaking at $\mathrm{pH} 6.5$ even if both macromolecules have charges 677 with the same sign. Charge regulation mechanism gives the 678 weaker attraction for $\mathrm{pH} 5$, as can be seen in Figure 5b. For $\mathrm{pH} 679$ $<4.5$ and $\mathrm{pH}>7$, no attractive interactions were registered. 680 Complexation for aggregates of 6INS (Figure 5c) resulted 681 much more intense than for 4INS aggregates, even though on 682 the same $\mathrm{pH}$ range, highlighting the importance of INS 683 molecules number forming the aggregates to be associated with 684 the polysaccharide.

685

From these sets of simulations, it can be presumed that 686 INS-CS interactions certainly depend on the number of INS $687 \mathrm{f} 6$ 
688 units. In fact, in Figure 6, it can be observed not only how the 689 complexation can be propitiated but also how this process also 690 adds intensity for an increasing INS number in the oligomeric 691 state.

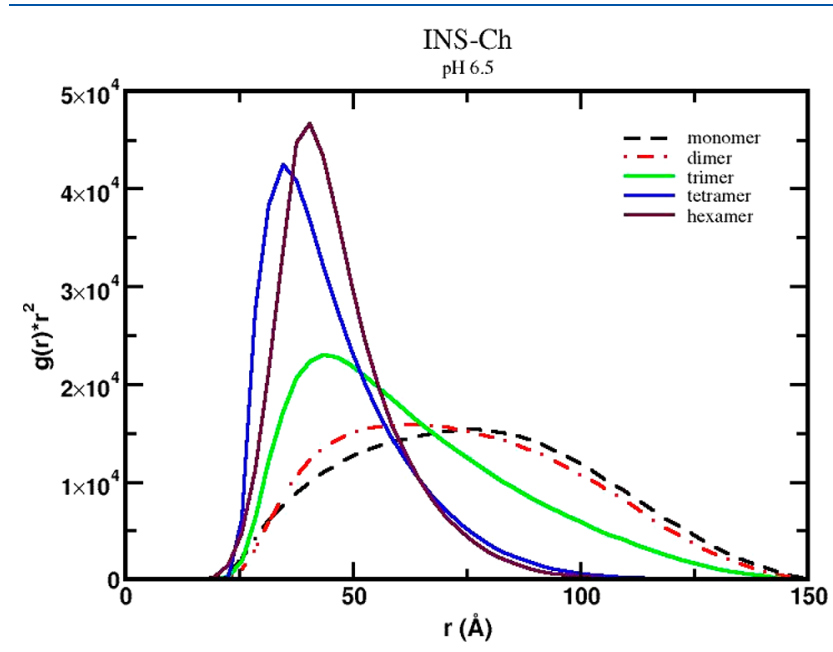

Figure 6. Computed free energy derivatives as a function of the separation distance between the protein center and the polyelectrolyte beads $[g(r) \cdot r 2]$, for different number of INS units at $\mathrm{pH}$ 6.5. Different lines represent each regime: solid (Coulombic attraction), dashed and dotted (attraction due the charge regulation mechanism), and dashed (Coulombic repulsion). The oligomeric state (from monomer to hexamer) is indicated in the plot.

692 It is important to highlight the fact that each simulation was 693 conducted considering a fixed number of INS units, a 694 condition maintained for the entire duration of the simulation 695 procedure. Meanwhile, the experimental results showed that 696 this number can vary as long as $\mathrm{pH}$ does. ${ }^{53}$ For the simulations, 697 is also important to note that when the number of INS 698 monomers is changed during the process, the $\mathrm{pH}$ range at 699 which the complexation occurs also changes, while the 700 experimental data shows that the complexation process is 701 favored at a fixed $\mathrm{pH}$ range.

702 Even more, it needs to be considered that both the protein 703 and the polysaccharide used in this point, i.e. simulation 704 approach, constitutes an oversimplification of a highly complex 705 real situation, and therefore some differences may appear while 706 comparing the simulated interaction $\mathrm{pHs}$ and those obtained 707 from experimental data. ${ }^{44}$ Nevertheless, this computational 708 approach provides a practical mean to rationalize the complex 709 interplay of the physical and chemical interactions involved in 710 the process.

711 The set of figures presented here suggests that CS's chain 712 interacts more efficiently, i.e. in a wider $\mathrm{pH}$ range, with INS 713 aggregates formed by the highest monomer number; these 714 results are in line with those experimentally obtained in ref 19. 715 As a result of considering one polymeric chain interacting with 716 one or several INS units, the comparison between simulations 717 and experimental findings become difficult, because the 718 experimental results indicate that multiple units of CS and 719 INS as well, are involved in the complete complexation 720 process. Furthermore, it has been described that both species 721 have $\mathrm{pH}$ dependent self-aggregation kinetics that cannot be 722 discarded, ${ }^{69-71}$ even though the concentrations used in this 723 work were low enough to minimize this effect. ${ }^{72}$

\section{CONCLUSIONS}

Experimental techniques proved the existence of macro- 724 molecular interactions between INS and CS, allowing us to 725 obtain quantitative parameters that characterize the complex- 726 ation process. Protein aggregation induced by the CS presence 727 was monitored by turbidimetric measurements and during 728 complexes formation. All the techniques combined allowed us 729 to determine that $\mathrm{pH} 6$ was the more appropriate level for 730 maximizing complex formation via electrostatic interactions. 731

The chemical groups involved in such interactions were 732 highlighted by FTIR, in dehydrated systems for which the 733 complexation had already occurred in aqueous solution. 734 Interactions between INS and CS were manifested by the 735 subtle displacement of peaks corresponding to amide I and 736 amide II in the formed complex.

737

Modeling data derived from the turbidimetric measurements 738 allowed to establish that, under a dilute regime, complexation 739 phenomenon proceeds rapidly, and the type of structure 740 formed is a cluster formed via bridging flocculation. 741

On the other hand, constant-pH Monte Carlo simulations 742 were performed in order to further elucidated INS-CS 743 interactions and complex formation. These simulations showed 744 that two parameters are the most relevant for the INS-CS 745 complexation process. On one hand, as the INS unit number 746 increases so does the complexation probability. On the other 747 hand, the $\mathrm{pH}$ should be higher than 5.5 for macromolecular 748 interactions, which correlates with $\zeta$-potential measurements, 749 indicating that the experimental $\mathrm{pH}$ range should be $5.5<\mathrm{pH} 750$ $<6.5$. This $\zeta$-potential range ensures the electrostatic 751 interactions between the two species.

752

In this context, the charge regulation mechanism can be 753 considered as a previous phase toward complexation or an 754 incipient complexation stage caused by weak interactions of 755 Coulombic nature. In any case, this mechanism should not be 756 ruled out, especially for $\mathrm{pHs}$ that render both species with the 757 same net charges or at $\mathrm{pH}$ values that are too close with 758 protein $\mathrm{pI}$. Simulations turned out to be a very interesting tool 759 to elucidate a $\mathrm{pH}$-induced mechanism that could translate into 760 attractive forces due to electrostatic interactions and repulsive 761 forces for situations in which the macromolecules have the 762 same surface charge.

These results are of extreme relevance, because $\mathrm{pH}$, and 764 therefore the INS aggregation state or the number of 765 aggregated monomers ( $n$ INS), must be carefully considered 766 for a complete process understanding. In this context these 767 results are in line with those published before in the work of 768 Silva et al. ${ }^{19}$

From a technological point of view, the knowledge of INS- 770 CS parameters that rule complex formation could be an 771 alternative for developing a new generation of drugs allowing 772 INS protection from the hostile conditions of the body and 773 increasing its absorption. In summary, these findings have basic 774 and practical impacts as they could be exploited to exert the 775 controlled release of INS in therapeutic formulations by using 776 CS based complexes.

\section{ASSOCIATED CONTENT}

\section{S Supporting Information}

The Supporting Information is available free of charge at 780 https://pubs.acs.org/doi/10.1021/acs.jcim.9b00814. 781

Table S1 and Figure S1 (PDF) 


\section{AUTHOR INFORMATION}

\section{Corresponding Author}

785 *Email: flbarroso@usp.br.

786 ORCID $\odot$

${ }_{787}$ Cecilia Prudkin-Silva: 0000-0001-8593-845X

788 Oscar E. Pérez: 0000-0002-8223-1077

789 Karina D. Martínez: 0000-0002-5208-6067

790 Fernando L. Barroso da Silva: 0000-0003-2526-2085

791 Notes

792 The authors declare no competing financial interest.

\section{ACKNOWLEDGMENTS}

794 C.P.S., O.E.P., and K.D.M. thank Universidad de Buenos Aires 795 [Project 20020150100079BA]; Agencia Nacional de Promo796 ción Cientifica y Tecnológica (ANPCyT) [(PICT 2015-3866 797 and PICT2017-1683); and CONICET of Argentina for 798 support. F.L.B. thanks the Fundação de Amparo à Pesquisa 799 do Estado de São Paulo [Fapesp 2015/16116-3], the Conselho 800 Nacional de Desenvolvimento Cientifico e Tecnológico $801(\mathrm{CNPq})$, and the USP through the NAP-CatSinQ (Research 802 Core in Catalysis and Chemical Synthesis) for support and 803 Rice University for computing hours through the international 804 collaboration program with USP and at The Swedish National 805 Infrastructure for Computing (SNIC 2019/1-32) where part of 806 the simulations were performed.

\section{REFERENCES}

808 (1) Kocak, G.; Tuncer, C.; Bütün, V. PH-Responsive Polymers. 809 Polym. Chem. 2017, 8 (1), 144-176.

810 (2) Wagoner, T. B.; Foegeding, E. A. Whey Protein-Pectin Soluble 811 Complexes for Beverage Applications. Food Hydrocolloids 2017, 63, 812 130-138.

813 (3) Feng, Z.; Zhang, T.; Wang, H.; Xu, B. Supramolecular Catalysis 814 and Dynamic Assemblies for Medicine. Chem. Soc. Rev. 2017, 46 (21), $8156470-6479$.

816 (4) Mendes, A. C.; Baran, E. T.; Reis, R. L.; Azevedo, H. S. Self817 Assembly in Nature: Using the Principles of Nature to Create 818 Complex Nanobiomaterials. Wiley Interdiscip. Rev. Nanomedicine 819 Nanobiotechnology 2013, 5 (6), 582-612.

820 (5) Smirnova, E.; Safenkova, I.; Stein-Margolina, V.; Shubin, V.; 821 Polshakov, V.; Gurvits, B. PH-Responsive Modulation of Insulin 822 Aggregation and Structural Transformation of the Aggregates. 823 Biochimie 2015, 109 (December), 49-59.

824 (6) Palao-Suay, R.; Gómez-Mascaraque, L. G.; Aguilar, M. R.; 825 Vázquez-Lasa, B.; Román, J. S. Self-Assembling Polymer Systems for 826 Advanced Treatment of Cancer and Inflammation. Prog. Polym. Sci. 827 2016, 53, 207-248.

828 (7) Fernández-Urrusuno, R.; Calvo, P.; Remuñán-López, C. 829 Enhancement of Nasal Absorption of Insulin Using Chitosan 830 Nanoparticles. Pharm. Res. 1999, 16 (10), 1576.

831 (8) Ma, Z.; Yeoh, H. H.; Lim, L. Y. Formulation PH Modulates the 832 Interaction of Insulin with Chitosan Nanoparticles. J. Pharm. Sci. 833 2002, 91 (6), 1396-1404.

834 (9) He, Z.; Santos, J. L.; Tian, H.; Huang, H.; Hu, Y.; Liu, L.; Leong, 835 K. W.; Chen, Y.; Mao, H. Q. Scalable Fabrication of Size-Controlled 836 Chitosan Nanoparticles for Oral Delivery of Insulin. Biomaterials 837 2017, 130, 28-41.

838 (10) Gatti, T. H. H.; Eloy, J. O.; Ferreira, L. M. B.; Da Silva, I. C.; 839 Pavan, F. R.; Gremião, M. P. D.; Chorilli, M. Insulin-Loaded 840 Polymeric Mucoadhesive Nanoparticles: Development, Character841 ization and Cytotoxicity Evaluation. Brazilian J. Pharm. Sci. 2018, 54 842 (1), $1-10$

843 (11) Roglic, G.; Norris, S. L. Guidelines on Second-and Third-Line 844 Medicines and Type of Insulin for the Control of Blood Glucose
Levels in Non-Pregnant Adults with Diabetes Mellitus. Ann. Intern. 845 Med. 2018, 169, 394-397.

(12) Naha, P.; Kanchan, V.; Manna, P. K.; Panda, A. K. Improved 847 Bioavailability of Orally Delivered Insulin Using Eudragit-L30D 848 Coated PLGA Microparticles. J. Microencapsulation 2008, 25 (4), 849 248-256.

850

(13) Sharma, S.; Jyoti, K.; Sinha, R.; Katyal, A.; Jain, U. K.; Madan, J. 851 Protamine Coated Proliposomes of Recombinant Human Insulin Encased 852 in Eudragit S100 Coated Capsule Offered Improved Peptide Delivery and 853 Permeation across Caco-2 Cells; Elsevier B.V., 2016; Vol. 67. (14) Shukla, S. K.; Mishra, A. K.; Arotiba, O. A.; Mamba, B. B. 855 Chitosan-Based Nanomaterials: A State-of-the-Art Review. Int. J. Biol. 856 Macromol. 2013, 59, 46-58.

(15) Kaur, S.; Dhillon, G. S. The Versatile Biopolymer Chitosan: 858 Potential Sources, Evaluation of Extraction Methods and Applications. 859 Crit. Rev. Microbiol. 2014, 40 (2), 155-175.

(16) Shalaby, T. I.; El-Refaie, W. M. Bioadhesive Chitosan-Coated 861 Cationic Nanoliposomes With Improved Insulin Encapsulation and 862 Prolonged Oral Hypoglycemic Effect in Diabetic Mice; American 863 Pharmacists Association, 2018; Vol. 107.

(17) Al-Remawi, M.; Elsayed, A.; Maghrabi, I.; Hamaidi, M.; Jaber, 865 N. Chitosan/Lecithin Liposomal Nanovesicles as an Oral Insulin 866 Delivery System. Pharm. Dev. Technol. 2017, 22 (3), 390-398. 867

(18) Sadhasivam, L.; Dey, N.; Francis, A. P.; Devasena, T. 868 Transdermal Patches of Chitosan Nanoparticles for Insulin Delivery. 869 Int. J. Pharm. Pharm. Sci. 2015, 7 (5), 84-88.

(19) Silva, C. P.; Martinez, J. H.; Martinez, K. D.; Farias, M. E.; 871 Leskow, F. C.; Perez, O. E. Proposed Molecular Model for 872 Electrostatic Interactions between Insulin and Chitosan. Nano- 873 Complexation and Activity in Cultured Cells. Colloids Surf., A 2018, 874 537, 425-434.

(20) Gaul, R.; Ramsey, J. M.; Heise, A.; Cryan, S.-A.; Greene, C. M. 876 Nanotechnology Approaches to Pulmonary Drug Delivery: Targeted 877 Delivery of Small Molecule and Gene-Based Therapeutics to the Lung; 878 Elsevier Inc., 2018.

(21) Gedawy, A.; Martinez, J.; Al-Salami, H.; Dass, C. R. Oral 880 Insulin Delivery: Existing Barriers and Current Counter-Strategies. J. 881 Pharm. Pharmacol. 2018, 70 (2), 197-213.

882

(22) Khafagy, E. S.; Morishita, M.; Onuki, Y.; Takayama, K. Current 883 Challenges in Non-Invasive Insulin Delivery Systems: A Comparative 884 Review. Adv. Drug Delivery Rev. 2007, 59 (15), 1521-1546. 885

(23) Ichikawa, S.; Iwamoto, S.; Watanabe, J. Formation of 886 Biocompatible Nanoparticles by Self-Assembly of Enzymatic Hydro- 887 lysates of Chitosan and Carboxymethyl Cellulose. Biosci., Biotechnol., 888 Biochem. 2005, 69 (9), 1637-1642.

(24) Jönsson, B.; Lund, M.; Barroso Da Silva, F. L. Electrostatics in 890 Macro-Molecular Solutions. In Food Colloids: Self-Assembly and 891 Material Science; Dickinson, E., Leser, M. E., Eds.; Royal Society of 892 Chemistry: Cambridge, 2007; p 129.

(25) da Silva, F. L. B.; Jonsson, B. Polyelectrolyte-Protein 894 Complexation Driven by Charge Regulation. Soft Matter 2009, 5895 (15), 2862. 896

(26) Verwey, E. J. W.; Overbeek, J. T. G.; Overbeek, J. T. G. Theory 897 of the Stability of Lyophobic Colloids; Courier Corporation, 1999. 898

(27) Kirkwood, J. G.; Shumaker, J. B. Forces between Protein 899 Molecules in Solution Arising from Fluctuations in Proton Charge 900 and Configuration. Proc. Natl. Acad. Sci. U. S. A. 1952, 38 (10), 863- 901 871.

(28) De Vos, W. M.; Biesheuvel, P. M.; De Keizer, A.; Kleijn, J. M.; 903 Stuart, M. A. C. Adsorption of the Protein Bovine Serum Albumin in 904 a Planar Poly(Acrylic Acid) Brush Layer as Measured by Optical 905 Reflectometry. Langmuir 2008, 24 (13), 6575-6584.

(29) Lund, M.; Jönsson, B. Charge Regulation in Biomolecular 907 Solution. Q. Rev. Biophys. 2013, 46 (3), 265-281.

(30) Barroso Da Silva, F. L.; Boström, M.; Persson, C. Effect of 909 Charge Regulation and Ion-Dipole Interactions on the Selectivity of 910 Protein-Nanoparticle Binding. Langmuir 2014, 30 (14), 4078-4083. 911 
912 (31) De Kruif, C. G.; Weinbreck, F.; De Vries, R. Complex 913 Coacervation of Proteins and Anionic Polysaccharides. Curr. Opin. 914 Colloid Interface Sci. 2004, 9 (5), 340-349.

915 (32) Srivastava, D.; Santiso, E.; Gubbins, K.; Barroso Da Silva, F. L. 916 Computationally Mapping PKaShifts Due to the Presence of a 917 Polyelectrolyte Chain around Whey Proteins. Langmuir 2017, 33 918 (42), 11417-11428.

919 (33) Li, J.; Wu, Y.; Zhao, L. Antibacterial Activity and Mechanism of 920 Chitosan with Ultra High Molecular Weight. Carbohydr. Polym. 2016, 921 148, 200-205.

922 (34) Tang, D. W.; Yu, S. H.; Ho, Y. C.; Huang, B. Q.; Tsai, G. J.; 923 Hsieh, H. Y.; Sung, H. W.; Mi, F. L. Characterization of Tea 924 Catechins-Loaded Nanoparticles Prepared from Chitosan and an 925 Edible Polypeptide. Food Hydrocolloids 2013, 30 (1), 33-41.

926 (35) Weijers, M.; Van De Velde, F.; Stijnman, A.; Van De 927 Pijpekamp, A.; Visschers, R. W. Structure and Rheological Properties 928 of Acid-Induced Egg White Protein Gels. Food Hydrocolloids 2006, 20 929 (2-3), 146-159.

930 (36) Perez, O.; Haros, M.; Suarez, C. Corn Steeping: Inuence of 931 Time and Lactic Acid on Isolation and Thermal Properties of Starch. 932 J. Food Eng. 2001, 48 (10), 251-256.

933 (37) Barroso da Silva, F. L.; Lund, M.; Jönsson, B.; Åkesson, T. On 934 the Complexation of Proteins and Polyelectrolytes. J. Phys. Chem. B 935 2006, 110 (9), 4459-4464.

936 (38) Barroso da Silva, F. L.; Dias, L. G. Development of Constant$937 \mathrm{PH}$ Simulation Methods in Implicit Solvent and Applications in 938 Biomolecular Systems. Biophys. Rev. 2017, 9 (5), 699-728.

939 (39) Baek, M.; Park, T.; Heo, L.; Park, C.; Seok, C. Galaxy 940 Homomer: A Web Server for Protein Homo-Oligomer Structure 941 Prediction from a Monomer Sequence or Structure. Nucleic Acids Res. 942 2017, 45 (W1), W320-W324.

943 (40) Jönsson, B.; Wennerstrom, H. Thermodynamics of Ionic 944 Amphiphile-Water Systems. J. Colloid Interface Sci. 1981, 80 (2), 482945496.

946 (41) Olatunji, O. Natural Polymers: Industry Techniques and 947 Applications; Springer International Publishing: Switzerland, 2016.

948 (42) Levesque, D.; Weis, J.; Hansen, J. Monte Carlo Methods in 949 Statistical Physics; Binder, K., Ed.; Springer-Verlag: Berlin, 1986.

950 (43) Barroso Da Silva, F. L. Peculiaridades Nos Mecanismos 951 Moleculares de Protéinas Em Solução Aquosa: Exemplo Da Importância 952 Do Equilibrio Ácido-Base Para Aplicações Em Biotecnologia; 2013; Vol. 953131.

954 (44) Montellano Duran, N.; Spelzini, D.; Wayllace, N.; Boeris, V.; 955 Barroso da Silva, F. L. A Combined Experimental and Molecular 956 Simulation Study of Factors Influencing Interaction of Quinoa 957 Proteins-Carrageenan. Int. J. Biol. Macromol. 2018, 107, 949-956.

958 (45) Frenkel, D.; Smit, B. Understanding Molecular Simulation: From 959 Algorithms to Applications; Academic Press: San Diego, 1996.

960 (46) Engkvist, O.; Karlström, G. A Method to Calculate the 961 Probability Distribution for Systems with Large Energy Barriers. 962 Chem. Phys. 1996, 213 (1-3), 63-76.

963 (47) Montellano Duran, N. Propiedades Estructurales Y Funcionales 964 de Las Proteinas de Chenopodium Quinoa y Sus Hidrolizados. Univ. 965 Nac. Rosario 2017, 1-11.

966 (48) Whittingham, J. L.; Scott, D. J.; Chance, K.; Wilson, A.; Finch, 967 J.; Brange, J.; Dodson, G. G. Insulin at PH 2: Structural Analysis of 968 the Conditions Promoting Insulin Fibre Formation. J. Mol. Biol. 2002, 969318 (02), 479-490.

970 (49) Tanford, C.; Epstein, J. The Physical Chemistry of Insulin. I. 971 Hydrogen Ion Titration Curve of Zinc-Free Insulin. J. Am. Chem. Soc. 972 1954, 76 (8), 2163-2169.

973 (50) Porath, J.; Hao Li, C. Elution and Displacement Analysis of 974 Insulin and Adrenocorticotropic Peptides on Pre-Treated Carbon. 975 Biochim. Biophys. Acta 1954, 13, 268-277.

976 (51) Wintersteiner, O.; Abramson, H. The Isoelectric Point of 977 Insulin. Electrical Properties of Adsorbed and Crystalline Insulin. J. 978 Biol. Chem. 1933, 99 (3), 741-753.

979 (52) Righetti, P. G.; Caravaggio, T. Isoelectric Points and Molecular 980 Weights of Proteins: A Table. J. Chromatogr. A 1976, 127 (1), 1-28.
(53) Dunn, M. F. Zinc-Ligand Interactions Modulate Assembly and 981 Stability of the Insulin Hexamer-a Review. BioMetals 2005, 18, 295- 982 303.

(54) Stirpe, A.; Pantusa, M.; Rizzuti, B.; Sportelli, L.; Bartucci, R.; 984 Guzzi, R. Early Stage Aggregation of Human Serum Albumin in the 985 Presence of Metal Ions. Int. J. Biol. Macromol. 2011, 49 (3), 337-342. 986

(55) Holthauzen, L. M. F.; Auton, M.; Sinev, M.; Rösgen, J. Protein 987 Stability in the Presence of Cosolutes. Methods Enzymol. 2011, 492, 988 61.

989

(56) Juarez, J.; Lopez, S. G.; Cambon, A.; Taboada, P.; Mosquera, V. 990 Influence of Electrostatic Interactions on the Fibrillation Process of 991 Human Serum Albumin. J. Phys. Chem. B 2009, 113 (30), 10521- 992 10529.

993

(57) Matalanis, A.; Jones, O. G.; McClements, D. J. Structured 994 Biopolymer-Based Delivery Systems for Encapsulation, Protection, 995 and Release of Lipophilic Compounds. Food Hydrocolloids 2011, 25996 (8), 1865-1880.

997

(58) Mukhopadhyay, P.; Sarkar, K.; Chakraborty, M.; Bhattacharya, 998 S.; Mishra, R.; Kundu, P. P. Oral Insulin Delivery by Self-Assembled 999 Chitosan Nanoparticles: In Vitro and in Vivo Studies in Diabetic 1000 Animal Model. Mater. Sci. Eng., C 2013, 33 (1), 376-382. 1001 (59) Guo, L.; Liu, G.; Hong, R. Y.; Li, H. Z. Preparation and 1002 Characterization of Chitosan Poly(Acrylic Acid) Magnetic Micro- 1003 spheres. Mar. Drugs 2010, 8 (7), 2212-2222. 1004

(60) Alhosseini, S. N.; Moztarzadeh, F.; Mozafari, M.; Asgari, S.; 1005 Dodel, M.; Samadikuchaksaraei, A.; Kargozar, S.; Jalali, N. Synthesis 1006 and Characterization of Electrospun Polyvinyl Alcohol Nanofibrous 1007 Scaffolds Modified by Blending with Chitosan for Neural Tissue 1008 Engineering. Int. J. Nanomed. 2012, 7, 25-34. 1009

(61) Venkatesham, M.; Ayodhya, D.; Madhusudhan, A.; Veera Babu, 1010 N.; Veerabhadram, G. A Novel Green One-Step Synthesis of Silver 1011 Nanoparticles Using Chitosan: Catalytic Activity and Antimicrobial 1012 Studies. Appl. Nanosci. 2014, 4 (1), 113-119.

1013

(62) Heuser, M.; Cárdenas, G. Chitosan-Copper Paint Types as 1014 Antifouling. J. Chil. Chem. Soc. 2014, 59 (2), 2415-2419. 1015

(63) Prusty, A. K.; Sahu, S. K. Development and Evaluation of 1016 Insulin Incorporated Nanoparticles for Oral Administration. ISRN 1017 Nanotechnol. 2013, 2013, 1-6.

1018

(64) Sarmento, B.; Ferreira, D.; Veiga, F.; Ribeiro, A. Character- 1019 ization of Insulin-Loaded Alginate Nanoparticles Produced by 1020 Ionotropic Pre-Gelation through DSC and FTIR Studies. Carbohydr. 1021 Polym. 2006, 66 (1), 1-7.

1022

(65) Xu, Y.; Mazzawi, M.; Chen, K.; Sun, L.; Dubin, P. L. Protein 1023 Purification by Polyelectrolyte Coacervation: Influence of Protein 1024 Charge Anisotropy on Selectivity. Biomacromolecules 2011, 12 (5), 1025 $1512-1522$

1026

(66) Chen, K.; Xu, Y.; Rana, S.; Miranda, O. R.; Dubin, P. L.; 1027 Rotello, V. M.; Sun, L.; Guo, X. Electrostatic Selectivity in Protein- 1028 Nanoparticle Interactions. Biomacromolecules 2011, 12 (7), 2552- 1029 2561.

1030

(67) Xu, X.; Angioletti-Uberti, S.; Lu, Y.; Dzubiella, J.; Ballauff, M. 1031 Interaction of Proteins with Polyelectrolytes: A Comparison between 1032 Theory and Experiment. Langmuir 2019, 35, 5373.

(68) Wang, X.; Zheng, K.; Si, Y.; Guo, X.; Xu, Y. Protein- 1034 Polyelectrolyte Interaction: Thermodynamic Analysis Based on the 1035 Titration Method. Polymers (Basel, Switz.) 2019, 11, 82. 1036

(69) Huang, Y.; Lapitsky, Y. On the Kinetics of Chitosan/ 1037 Tripolyphosphate Micro- and Nanogel Aggregation and Their Effects 1038 on Particle Polydispersity. J. Colloid Interface Sci. 2017, 486, 27-37. 1039

(70) Baram, M.; Gilead, S.; Gazit, E.; Miller, Y. Mechanistic 1040 Perspective and Functional Activity of Insulin in Amylin Aggregation. 1041 Chem. Sci. 2018, 9 (18), 4244-4252.

1042

(71) Chung, L. H. C.; Birch, D. J. S.; Vyshemirsky, V.; Ryadnov, M. 1043 G.; Rolinski, O. J. Insulin Aggregation Tracked by Its Intrinsic TRES. 1044 Appl. Phys. Lett. 2017, 111 (26), 263701.

1045

(72) Philippova, O. E.; Volkov, E. V.; Sitnikova, N. L.; Khokhlov, A. 1046 R.; Desbrieres, J.; Rinaudo, M. Two Types of Hydrophobic 1047 Aggregates in Aqueous Solutions of Chitosan and Its Hydrophobic 1048 Derivative. Biomacromolecules 2001, 2 (2), 483-490. 
1050 (73) https://pubchem.ncbi.nlm.nih.gov/compound/16129672 (ac1051 cessed Dec 13, 2018). 